\title{
Comparison of Cryo TEM Images Obtained with Zernike and Hole-Free Phase Plates
}

\author{
Naoki Hosogi ${ }^{1}$,Anindito Sen $^{1}$ and Hirohumi Iijima ${ }^{1}$ \\ 1. EM Business Unit, JEOL Ltd., Akishima, Japan
}

Cryo transmission electron microscopy (TEM) provides structural information of a specimen close to its natural state without any disturbance due to the specimen preparation process. The cryo specimens exhibit low contrast in TEM images even using large defocus phase contrast imaging. Cryo TEM is more advantageous when it is combined with a technique of contrast enhancement using a phase plate.

Several applications of a Zernike phase plate (ZPP) were already reported $[1,2]$. The ZPP is made of a thin amorphous carbon film with a center hole whose diameter is less than $1 \mu \mathrm{m}$. The contrast enhancement by the ZPP can be achieved by phase shifting the scattered beam by half $\pi$ with respect to the unscattered direct beam. The half $\pi$ phase shift is given to the diffracted beams by the thin film of the phase plate placed at the back focal plane, while the direct unscattered electrons just pass through the center hole with no phase shift. However, characteristic fringes appeare around edges of high-contrast objects such as high density particles in the images with a ZPP [3, 4]. The fringe artifact results from the sudden jump of the contrast transfer function due to the sudden cut-on frequency of the phase plate hole. A "hole-free phase plate" (HFPP) [5] is considered to reduce the fringe artifact. The HFPP is a thin amorphous carbon film phase plate without hole. In the HFPP, the charging at a local area in the thin film, which is induced by the direct beam, acts as a tiny sized phase shifter to achieve a phase contrast. Diameter of this charging area is estimated to be several tens of $\mathrm{nm}$, which is pretty smaller than the center hole of the ZPP, when we use FEG for the electron source. Therefore, the fringe effect of HFPP is less than that of ZPP.

In the present study, we tried cryo TEM observations for some biological specimens with the ZPP and the HFPP. The ZPP and HFPP images (Fig. 1b and c) with a near-to-focus condition exhibited highly improved contrast compared to the largely defocused phase contrast image (Fig. 1a). Reduced fringe contrast is observed in a HFPP image shown in Fig. 1c, compared with a ZPC image shown in Fig. 1b. In addition to the reduction of the fringe contrast, the HFPP does not require any alignment procedure due to lack of a center hole. Thus, the data acquisition with HFPP could be done easier than that with ZPP. The high contrast HFPP images with the reduced fringe effect should lead to a higher-resolution observation of cryo specimens. 


\section{References}

[1] K Nagayama et al, Microsc. Today 18 (2010), p. 10.

[2] W Dai et al, Nature 502 (2013), p. 707.

[3] R Danev and K Nagayama, Biophysics 2 (2006), p. 35.

[4] Y Fukuda et al, J. Struct. Biol. 168 (2009), p. 476.

[5] M Malac et al, Ultramicroscopy 118 (2012), p. 77.

[6] Mr. N Tominaga (National Cancer Center Research Institute and TheUniversity of Tokyo) is thanked for kindly providing exosomes.
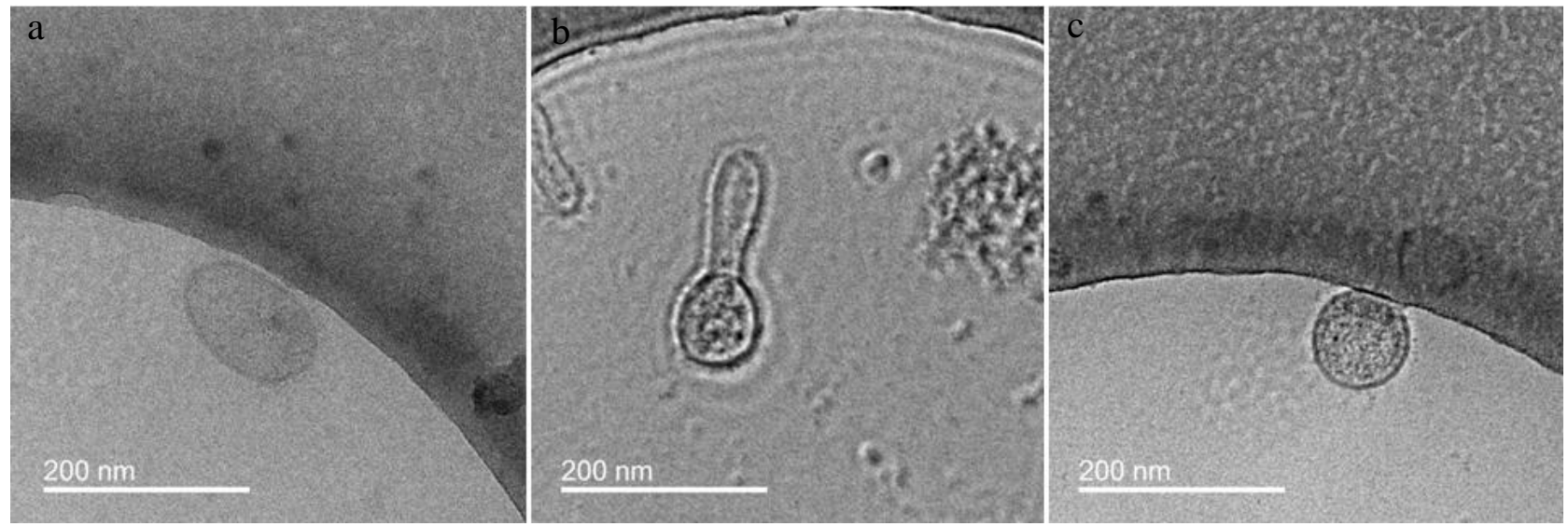

Figure 1. Cryo TEM images of ice-embedded exosomes acquired with a few microns of defocus (conventional) (a), ZPP (b) and HFPP (c). The image in B and C was taken at a near-to-focus condition. Scale bar: $200 \mathrm{~nm}$. 